\title{
Rankine-Merchant Approach to Specification of Flexural Buckling Coefficient for Fire Situation
}

\author{
Mariusz Maślak \\ Cracow University of Technology, Cracow 31-155, Poland
}

\begin{abstract}
An alternative design technique of the evaluation of flexural buckling coefficient determined for fire situation is presented and widely discussed in the present paper. The proposed methodology is based on the Rankine-Merchant concept. An equivalent buckling coefficient is adopted as the objective measure of the reduction of member fire resistance. It is specified as a product of classical coefficient which deals only with buckling phenomenon and the ratio of the reduction of steel yield point at fire temperature. Some calculation formulae, differentiated in relation to model complexity, are recommended to reliably assess the value of searched coefficient. Obtained results are compared with adequate buckling factors resulting from typical standard analysis. Moreover, a quantitative comparison is shown here, in which the influence on conclusive resistance of some factors being the indirect effect of the consideration of imperfect structural members is examined in detail.
\end{abstract}

Key words: Buckling coefficient, Rankine-Merchant approach, fire situation.

\section{Introduction}

The Rankine-Merchant approach is widely used design technique, helpful for the prediction of real failure load, especially if the resistance of framed structures is assessed. It is based on the independent and separate analyses of the strength of considered member and its stability. Determination of the strength deals with the evaluation of squashing or yielding stresses, then pure plastic behaviour of structural element is taken into account in this case. On the other hand, for stability analysis, the pure elastic buckling load is looked for. Consequently, simple assumption about the way of the interaction between those two failure modes is made. The main advantage of such methodology is its simplicity. Furthermore, one can say that the results obtained by this approach seem to be sufficiently reliable because of its multiple experimental verification. Let us notice that the history of succesfull application of this formulation is significantly long. Based on the empirical formula given by W.J.M. Rankine in 1866 for

Corresponding author: Mariusz Maslak, Dr., professor, research fields: steel structures and fire engineering. E-mail: mmaslak@pk.edu.pl. perfectly straight columns, W. Merchant in 1954 developed a more generalized theory to determine the failure load factor of rigidly jointed frames, subjected to proportional loading.

In classical analysis in which the Rankine-Merchant approach is considered, the failure load of load-bearing structure is estimated for proportionally increasing external loads but for members remaining at ambient temperature. Extending such examination to the case of fully developed fire requires incorporation to typical design procedure some thermal effects resulting directly from the appearance of significantly high temperature of structural steel. Detailed investigations in this field were recently carried out by the group of scientists from Nanyang University in Singapore. Particularly W. S. Toh, K. H. Tan and T. C. Fung in Ref. [1], C. Y. Tang, K. H. Tan and S. K. Ting in Ref. [2] and Z. H. Huang and K. H. Tan in Ref. [3] have made an attempt to propose the suitable interaction formulae, adapted to single steel columns subject to fire. Those formulae have been further generalized by C.Y. Tang and K.H. Tan in Ref. [4] and by W. S. Toh, K. H. Tan and T. C. Fung in Ref. [5] to enable their application to the whole steel framed structures heated 
by fire. Moreover, the numerical studies about the accuracy of the proposed design methodology were undertaken by W. S. Toh, K. H. Tan and T. C. Fung in Ref. [6]. Besides those works the problem how to correctly estimate the rigid-plastic collapse load as well as the elastic critical load for steel framed structures for fire conditions has been studied also in Poland by W. Skowroński, even before the research conducted in Sigapore $[7,8]$.

The main aim of the present paper is making a quantitative comparison between the evaluations of failure loads which can be obtained by means of the application of Rankine-Merchant design procedure and corresponding results being the solution of traditional standard approach, recommended in PN-EN 1993-1-2 [9]. Considering the fact that in such standard calculation technique member fire resistance is estimated owing to the specification of special flexural buckling coefficient $\quad \chi_{\min , f, \Theta}=\min \left(\chi_{y, f, \theta}, \chi_{z, f, \Theta}\right)$, analogous coefficient is defined by the author in relation to the methodology proposed by $\mathrm{W}$. Merchant and generalized to the case of fully developed fire (symbol $\Theta\left[{ }^{\circ} \mathrm{C}\right]$ means here the temperature of analysed structural member).

\section{Standard Flexural Buckling Coefficient for Fire Situation}

The design fire resistance of uniformly heated and axially compressed steel member, assessed regarding to the elastic-plastic buckling instability failure mode, is usually determined [9] by means of the following formula:

$$
N_{b, f, \Theta, R d}=\chi_{\min , f, \Theta} A k_{y, \Theta} \frac{f_{y}}{\gamma_{M, f i}}
$$

where, $A\left[\mathrm{~m}^{2}\right]$ - area of member cross-section, $f_{y}[\mathrm{MPa}]$ - steel yield point established at ambient temperature (it is assumed that this is the room temperature $\left.\Theta=20^{\circ} \mathrm{C}\right), k_{y, \Theta}=f_{y, \Theta} / f_{y}$ - ratio of the reduction of steel yield point in fire (its values for particular steel temperatures are given in [9], $\gamma_{M, f i}-$ partial safety factor specified especially for fire situation (it is recommended to use $\gamma_{M, f i}=1,0$ ) .

In such equation the flexural buckling coefficient depends on the actual steel temperature $\Theta$ :

$$
\chi_{\min , f, \Theta}=\frac{1}{\varphi_{\max , \Theta}+\sqrt{\varphi_{\max , \Theta}^{2}-\bar{\lambda}_{\max , \Theta}^{2}}}
$$

where,

$$
\varphi_{\max , \Theta}=0,5\left(1+\alpha \bar{\lambda}_{\max , \Theta}+\bar{\lambda}_{\max , \Theta}^{2}\right) \text { and } \alpha=0,65 \sqrt{\frac{235}{f_{y}}}
$$

Moreover, the non-dimensional (normalized) slenderness $\bar{\lambda}_{\theta}$ is also a function of this temperature according to the relation:

$$
\bar{\lambda}_{\theta}=\bar{\lambda} \sqrt{\frac{k_{y, \theta}}{k_{E, \theta}}}
$$

where, $\bar{\lambda}-$ analogous non-dimensional member slenderness but calculated for the case of ambient temperature, $k_{E, \Theta}=E_{\Theta} / E-$ ratio of the reduction of Young modulus at fire temperature (its values determined for particular steel temperatures are also given in Ref. [9].

Let all notice that the imperfection factor $\alpha$ corresponding to fire situation does not depend on the shape of member cross-section so only one type of a buckling curve is then specified. This is a fundamental difference in relation to classical calculations, made for members remaining at ambient temperature, in which various buckling curves ( $a, b, c$ and $d$ ) are defined. The concept of the specification of a single buckling curve when fire conditions are considered is widely verified by experiments. It is significantly important that such "fire" buckling curve gives evaluations of the coefficients $x_{f i}$ which are much more conservative than suitable assessments of the coefficients $x$, determined for the case of room temperature. Such relation is well noticeable in Fig. 1, in which the comparison is made between the coefficients $\chi=\chi(\bar{\lambda})$ established for particular buckling curves specified for persistent design situation (without any considerations about fire threat) and the analogous coefficients $\chi_{f, 20}=\chi_{f, 20}(\bar{\lambda})$ calculated for fire situation, but when $\Theta=20^{\circ} \mathrm{C}$, i.e., for fire starting-point (at that time $\bar{\lambda}_{\theta}=\bar{\lambda}$ ). 


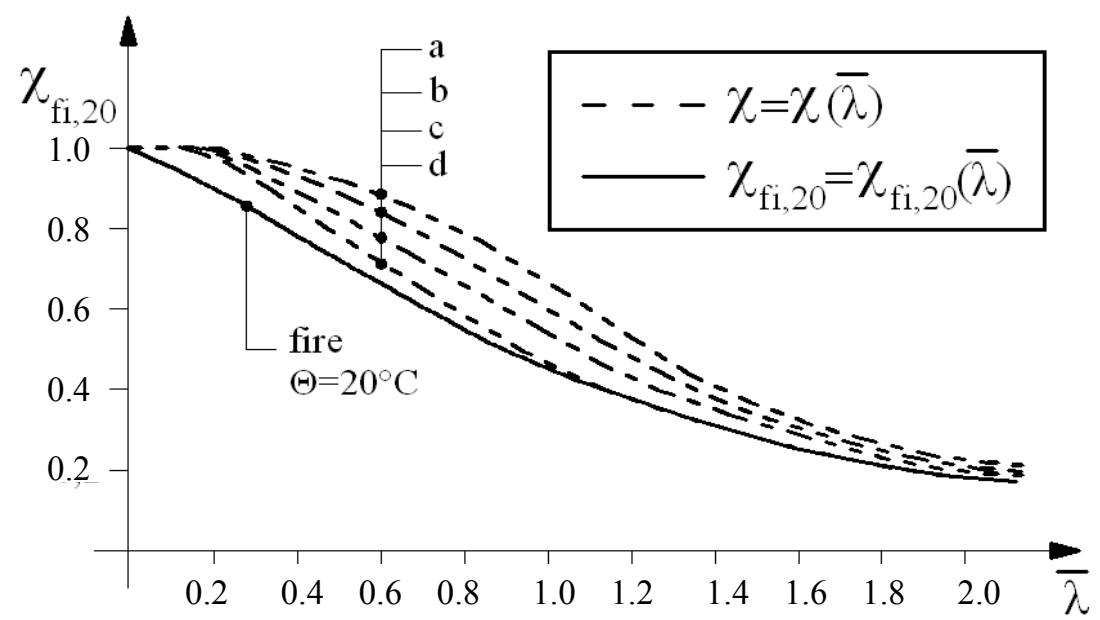

Fig. 1 Relations $\chi=\chi(\bar{\lambda})$ and $\chi_{f i, 20}=\chi_{f i, 20}(\bar{\lambda})$.

In Fig. 2 the influence of fire temperature $\Theta$ on reduction of design member fire resistance $N_{b, f, \Theta, R d}$ is shown in detail. Let all notice that, basing on Eq. (1), the following proportion is satisfied:

$$
\frac{N_{b, f i, Q R d}}{N_{b, f, 2,20, R d}}=\frac{\chi_{f i} k_{y, \Theta}}{\chi_{f, 20}}
$$

hence:

$$
N_{b, f i, \theta, R d}=\frac{\chi_{f i} k_{y, \Theta}}{\chi_{f i, 20}} N_{b, f i, 20, R d}
$$

Consequently, if the product $\chi_{f, \Theta}^{*}=\chi_{f i} k_{y, \Theta}$ means the equivalent flexural buckling coefficient for fire situation then its value can be a good measure of the reduction which is looked for. Its dependence on non-dimensional slenderness $\bar{\lambda}$ (not on the adequate slenderness $\bar{\lambda}_{\theta}$ ) is presented in Fig. 2 for chosen values of steel temperature $\Theta$.

\section{Specification of Buckling Coefficient for Fire Conditions with Respect to Rankine-Merchant Approach}

In general the interaction formula resulting from the Rankine-Merchant design concept has the following form (if $\gamma_{M, f i}=1,0$ then $N_{R M, f, R d}=N_{R M, f i}$ ):

$$
\frac{1}{N_{R M, f i}(\Theta)}=\frac{1}{N_{p l, f i}(\Theta)}+\frac{1}{N_{e l, f i}(\Theta)}
$$

where, $N_{p l, f i}$ is the rigid-plastic collapse load, $N_{e l, f i}$ is the elastic critical load, $N_{R M, f i}$ is the conclusive
Rankine-Merchant load.

The buckling coefficient $\chi_{f, \theta}$ is the measure of the reduction of conclusive member resistance in relation to its pure plastic carrying capacity, so:

$$
\chi_{f, \Theta}=\frac{N_{R M, f i}(\Theta)}{N_{p l, f i}(\Theta)}=\frac{N_{R M, f i}(\Theta)}{k_{y, \Theta} f_{y} A}
$$

Basing of such definition one can rearrange Eq. (7) to the form:

$$
\frac{1}{\chi_{f, \theta}}=1+\frac{k_{y, \theta} f_{y} A}{N_{e l, f i}}=1+\frac{k_{y, \theta} f_{y} A L_{e}^{2}}{\pi^{2} k_{E, \theta} E I}=1+\frac{k_{y, \theta} f_{y}}{\pi^{2} k_{E, \theta} E} \frac{L_{e}^{2}}{i^{2}}=1+\bar{\lambda}_{\theta}^{2}(9)
$$
where, $L_{e}$ is the buckling length of considered member, $I$ is the moment of inertia whereas $i$ is the radius of gyration, both calculated for the member cross section.

The value of buckling coefficient is then found as a simple reciprocal:

$$
\chi_{f, \theta}=\left(1+\bar{\lambda}_{\theta}^{2}\right)^{-1}
$$

Let us notice that the assessment of buckling factor $\chi_{f, \theta}$, obtained from Eq. (10), cannot be precise enough because the model of perfectly straight column was adopted to the calculation of elastic critical load. To receive its better evaluations at least the influence of geometrical imperfection should be additionally taken into cosideration. If the initial deflection of the column is assumed with its greatest value equal $e$ in the middle of the column height, then the interaction M-N must be 


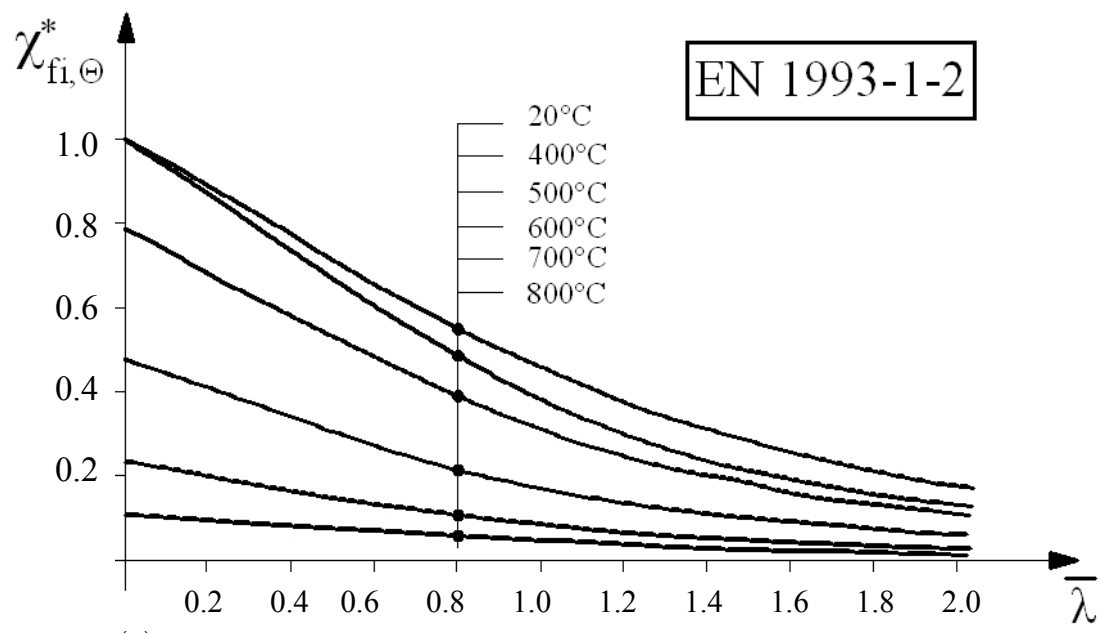

Fig. 2 Relations $\chi_{f, \Theta}^{*}=\chi_{f, \Theta}^{*}(\bar{\lambda})$.

studied as the effect of such imperfection. Let the simplest formula describing this interaction be adopted to the analysis:

$$
\frac{N_{p l, f i}}{k_{y, \theta} f_{y} A}+\frac{N_{p l, f} e}{k_{y, \theta} f_{y} W_{p l}}=1
$$

where, $W_{p l}$ is the plastic section modulus. It can be written in another form:

$$
\frac{N_{p l, \Theta}}{k_{y, \theta} f_{y} A}=\left(1+\frac{e A}{W_{p l}}\right)^{-1}
$$

Finally, substituting this formula to Eq. (9) in a place of the factor previously equal 1.0, the new equation for the assessment of flexural buckling coefficient $\chi_{f, \theta}$ may be proposed:

$$
\chi_{f, \theta}=\left(1+\frac{e A}{W_{p l}}+\bar{\lambda}_{\theta}^{2}\right)^{-1}
$$

It is noteworthy that adaptation to the analysis of slightly different interaction $\mathrm{M}-\mathrm{N}$ formula, giving much better approximation of obtained values to experimental results, described for $N_{p l, \Theta} /\left(k_{y, \Theta} f_{y} A\right) \geq 0,2$ in a form:

$$
\frac{N_{p l, \Theta}}{k_{y, \Theta} f_{y} A}+\frac{N_{p l, \Theta} e}{1,125 k_{y, \Theta} f_{y} W_{p l}}=1
$$

leads to analogous correction of considered buckling coefficient. It is now calculated by the equation:

$$
\chi_{f, \theta}=\left(1+\frac{e A}{1,125 W_{p l}}+\bar{\lambda}_{\theta}^{2}\right)^{-1}
$$

If the imperfect column is examined in the study then application of classical Euler formula for the assessment of $N_{e l, \Theta}$ critical load is not fully correct. More precise design technique is proposed to be used in [6]. Let the analysed column be simply supported with a pinned-roller joint at its top. Moreover, let this column be eccentrically-loaded with the parameter $\varepsilon$ being a measure of such eccentricity. In addition, the initial deflection of the member is also assumed with the value $e$ in the middle of the column height (see Fig. 3). By solving a second-order linear differential equation the following dependence is obtained:

$$
e=\varepsilon(\sec \beta-1)
$$

in which:

$$
\beta=\frac{\pi}{2} \sqrt{\frac{N_{e l, \Theta}}{N_{e l, \Theta}^{*}}}
$$

The value $N_{e l, \Theta}^{*}=\pi^{2} k_{E, \Theta} E I / L_{e}^{2}$ is in this formula the idealised Euler load. Substituting Eq. (17) to Eq. (16), after the rearrangement, the real value of $N_{e l, \Theta}$ can be expressed:

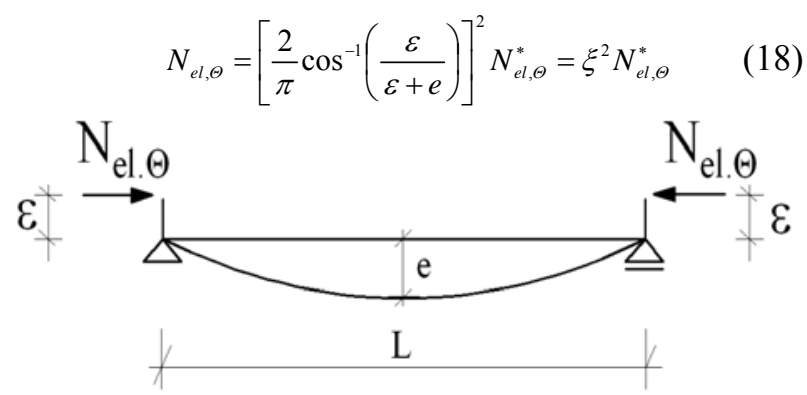

Fig. 3 Model of eccentrically-loaded column (according to [6]). 
So, basing on Eq. (18) the authors have the following proportion:

$$
\frac{k_{y, \Theta} f_{y} A}{N_{e l, \Theta}^{*}}=\frac{\bar{\lambda}_{\theta}^{2}}{\xi^{2}}
$$

which leads to more precise evaluation of buckling coefficient $\chi_{f, \theta}$ :

$$
\chi_{f, \Theta}=\left(1+\frac{e A}{1,125 W_{p l}}+\frac{\bar{\lambda}_{\theta}^{2}}{\xi^{2}}\right)^{-1}
$$

\section{Quantitative Analysis of Obtained Buckling Coefficients}

The comparison presented in this chapter deals with the equivalent buckling coefficients $\chi_{f, \Theta}^{*}=k_{y, \Theta} \chi_{f, \Theta}$. This factor can be considered as an objective measure of the reduction of member fire resistance because it includes not only the influence of buckling phenomenon but also the decreasing of material yield point at high temperature of constructional steel. At the beginning two kinds of coefficients $\chi_{f, \Theta}^{*}$ are compared in Table 1, in relation to the temperature $\Theta$ as well as to the non-dimensional slenderness $\bar{\lambda} \neq \bar{\lambda}_{\theta}$. First of those, marked by the symbol EN (which is the abbreviation of the word "euronorm"), is calculated by means of Eqs. (2) and (3), whereas the second, described as R-M (Rankine-Merchant), is the solution of Eq. (10). As the authors can see, evaluations of buckling coefficients made on the basis of the application of classical standard approach [9] are much more conservative than the other ones, being the result of using of Rankine-Merchant concept. However, as it has been said previously, formula (10) is not fully realistic. At that place the fact must be underlined that the parameter $k_{y, \Theta}=1.0$ only if $\Theta \leq 400^{\circ} \mathrm{C}$ but it is decreasing with increasing steel temperature for $\Theta>$ $400^{\circ} \mathrm{C}$. This is the reason of significant quantitative difference between the coefficients calculated for lower and higher temperature $\Theta$, both for "EN" and for "R-M" buckling coefficients.

To improve the accuracy of the assessment of such coefficients some initial geometrical imperfections were modelled and, as a result of this concept, the interaction $\mathrm{M}-\mathrm{N}$ was additionally taken into consideration. Two types of interaction formulae were examined (Eqs. (11) and (14)). Consequently, two equations were proposed for calculation of buckling coefficients (Eqs. (13) and (15)).

Let all see various buckling curves presented in Fig. 4. They are the solution of Eq. (13) for $\Theta=400^{\circ} \mathrm{C}$. The influence of imperfection magnitude on the shape of corresponding buckling curve is shown here. The ratio $(e A) / W_{p l}$ is the basic parameter differentiating the convexity of considered curves. Greater value of initial deflection $e$ is always connected with greater influence of bending in relation to axial compression, so simultaneously equivalent buckling coefficient must be decreasing. Another dependence is explained in Fig. 5. Presented buckling curves result from using Eq. (15) in which the parameter $(e A) / W_{p l}=0.2$ is assumed. As the

\begin{tabular}{|c|c|c|c|c|c|c|c|c|c|c|}
\hline \multirow[b]{2}{*}{$\bar{\lambda}$} & \multicolumn{2}{|c|}{$20^{\circ} \mathrm{C}$} & \multicolumn{2}{|c|}{$200^{\circ} \mathrm{C}$} & \multicolumn{2}{|c|}{$400^{\circ} \mathrm{C}$} & \multicolumn{2}{|c|}{$600^{\circ} \mathrm{C}$} & \multicolumn{2}{|c|}{$800^{\circ} \mathrm{C}$} \\
\hline & EN & R-M & $\mathrm{EN}$ & R-M & EN & R-M & EN & R-M & EN & R-M \\
\hline$\overline{0}$ & 1.000 & 1.000 & 1.000 & 1.000 & 1.000 & 1.000 & 0.470 & 0.470 & 0.110 & 0.110 \\
\hline 0.2 & 0.881 & 0.962 & 0.875 & 0.957 & 0.860 & 0.946 & 0.402 & 0.443 & 0.096 & 0.105 \\
\hline 0.4 & 0.771 & 0.862 & 0.759 & 0.849 & 0.728 & 0.814 & 0.338 & 0.378 & 0.082 & 0.092 \\
\hline 0.6 & 0.661 & 0.735 & 0.644 & 0.714 & 0.598 & 0.660 & 0.275 & 0.304 & 0.069 & 0.076 \\
\hline 0.8 & 0.554 & 0.610 & 0.532 & 0.584 & 0.476 & 0.552 & 0.205 & 0.239 & 0.056 & 0.062 \\
\hline 1.0 & 0.456 & 0.500 & 0.432 & 0.474 & 0.374 & 0.412 & 0.170 & 0.187 & 0.045 & 0.050 \\
\hline 1.2 & 0.373 & 0.410 & 0.349 & 0.385 & 0.296 & 0.327 & 0.133 & 0.148 & 0.036 & 0.040 \\
\hline 1.4 & 0.306 & 0.338 & 0.284 & 0.315 & 0.237 & 0.263 & 0.106 & 0.118 & 0.029 & 0.032 \\
\hline 1.6 & 0.253 & 0.281 & 0.234 & 0.260 & 0.192 & 0.215 & 0.086 & 0.096 & 0.024 & 0.027 \\
\hline 1.8 & 0.212 & 0.236 & 0.195 & 0.217 & 0.159 & 0.178 & 0.071 & 0.079 & 0.020 & 0.022 \\
\hline 2.0 & 0.179 & 0.200 & 0.164 & 0.184 & 0.133 & 0.149 & 0.060 & 0.067 & 0.017 & 0.019 \\
\hline
\end{tabular}

Table 1 Comparison between "EN" and “R-M" equivalent buckling coefficients. 
author can see, the equivalent buckling coefficient $\chi_{f, \Theta}^{*}$ is much lower with higher steel temperature $\Theta$.

Interesting effect can be observed based on the results presented in Table 2 for $\Theta=400^{\circ} \mathrm{C}$. More precise description of the interaction M-N leads to less conservative values of equivalent buckling coefficients, especially if the normalized slenderness $\bar{\lambda}$ is not high; however, difference between obtained outcomes is quite small.

In the next table (Table 3) the evaluations of equivalent buckling coefficients are collected, obtained for $\Theta=400^{\circ} \mathrm{C}$, owing to the application of Eq. (20) when $\xi=0.9$. They are compared with the other ones, resulting from Eq. (15). As it is shown here the influence of $\xi$ parameter is more significant, in particular for greater slenderness $\bar{\lambda}$.
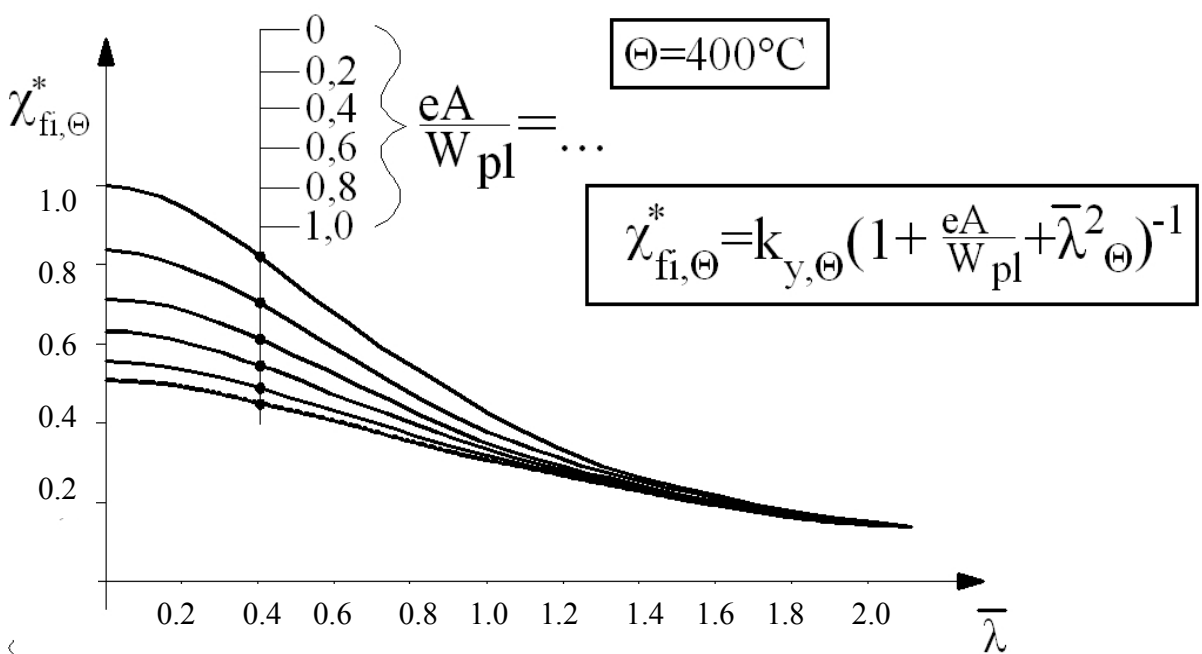

Fig. 4 Influence of initial imperfection $e$ on equivalent buckling coefficient.

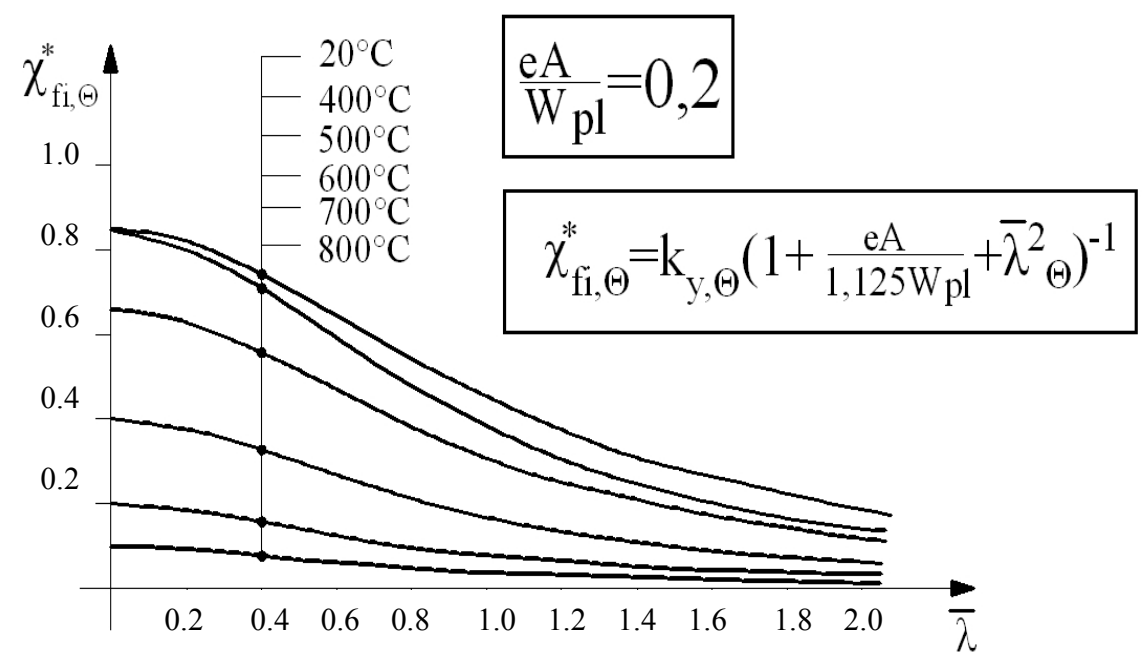

Fig. 5 Influence of steel temperature on equivalent buckling coefficient.

Table 2 Equivalent buckling coefficients, obtained for $\Theta=400^{\circ} \mathrm{C}$, based on various formulae describing interaction $\mathrm{M}-\mathrm{N}$.

\begin{tabular}{llllllllllll}
\hline $\bar{\lambda}$ & 0 & 0.2 & 0.4 & 0.6 & 0.8 & 1.0 & 1.2 & 1.4 & 1.6 & 1.8 & 2.0 \\
\hline Eq. (13) & 0.833 & 0.795 & 0.700 & 0.583 & 0.473 & 0.380 & 0.307 & 0.250 & 0.206 & 0.172 & 0.145 \\
Eq. (15) & 0.849 & 0.810 & 0.711 & 0.591 & 0.478 & 0.384 & 0.309 & 0.251 & 0.207 & 0.172 & 0.145 \\
\hline
\end{tabular}


Table 3 Values of equivalent buckling coefficients obtained for $\Theta=400^{\circ} \mathrm{C}$, based on the application Eq. (20) when $\xi=0.9$, in relation to the other ones, resulting from Eq. (15).

\begin{tabular}{|c|c|c|c|c|c|c|c|c|c|c|c|}
\hline $\bar{\lambda}$ & 0 & 0.2 & 0.4 & 0.6 & 0.8 & 1.0 & 1.2 & 1.4 & 1.6 & 1.8 & 2.0 \\
\hline Eq. (15) & 0.849 & 0.810 & 0.711 & 0.591 & 0.478 & 0.384 & 0.309 & 0.251 & 0.207 & 0.172 & 0.145 \\
\hline Eq. (20) if $\xi=0.9$ & 0.849 & 0.801 & 0.685 & 0.552 & 0.434 & 0.340 & 0.269 & 0.216 & 0.176 & 0.145 & 0.121 \\
\hline
\end{tabular}

\section{Summary and Conclusions}

The Rankine-Merchant approach to evaluation of real member resistance can be an interesting alternative in relation to classical standard methodology of such assessment, not only for the case of persistent design situation but also when accidental, fully-developed fire conditions are considered. In the present paper the equivalent flexural buckling coefficient $\chi_{f, \theta}^{*}$, which includes both influence of buckling phenomenon and the unfavourable effects of weakening of material properties being the result of high temperature, is adopted by the author as an objective measure of the reduction of examined resistance in fire. Some direct formulae are specified here to estimate the value of searched coefficient. They are different one from the other according to the ratio of their complexity, which corresponds with the various precision of determination of considered coefficient.

The quantitative comparison between the obtained outcomes leads to the conclusion that the coefficients calculated basing on the application of the standard approach are, as a rule, more conservative than adequate factors, being a results of using of Rankine-Merchant concept. Equivalent buckling coefficients, resulting from the simplest formula proposed in this article (Eq. (10)), would be able to be compared with the standard ones if typical multiple buckling curves, specified for calculations made at room temperature, were taken into account also in fire situation (Fig. 1). The concept that only a simple and very strong buckling curve is recommended in such case allows to include structural analysis made for fire situation not only the model uncertainty but also a great random variability of the parameters of potential fire. Besides that such simplification gives evaluations of searched coefficients which are safe in general. This statement is widely verified by experiments. On the other hand, all influences which are the effects of randomness of basic parameters describing a fire could be taken into consideration in another calculation phase, for example with the specification of suitable partial safety factors and/or with the adoption of accurate regula of load combination.

Using of Rankine-Merchant design approach gives the opportunity to judge the importance of various factors being the result of the analysis of members which are not perfect in statical sense. All those effects can be modelled owing to the application of the equivalent imperfection concept. Such imperfections can be adopted exemplarily as the initial member deflection or as the load eccentricity. As one can see in the presented paper the importance of those effects is frequently very significant. Let all notice that such imperfections are also considered in classical standard evaluations of suitable buckling coefficients, in which the Perry-Robertson design model is used, but their effects cannot be extracted in such simple and illustrative way because the formal structure of adequate calculation formula (see Eq. (2)) is more involved.

\section{References}

[1] W. S. Toh, K. H. Tan and T. C. Fung, Compressive resistance of steel columns in fire: Rankine approach, Journal of Structural Engineering 126 (3) (2000) 398-404.

[2] C. Y. Tang, K. H. Tan and S. K. Ting, Basis and application of a simple interaction formula for steel columns under fire conditions, Journal of Structural Engineering 127 (10) (2001) 1206-1213.

[3] Z. F. Huang and K. H. Tan, Rankine approach for fire resistance of axially-restrained steel column, in: Proceedings of 4th International Conference on Performance-Based Codes and Fire Safety Design Methods, Melbourne, Australia, 2002, pp. 225-234. 
[4] C. Y. Tang and K. H. Tan, Basis and applications of simple application formula for steel frames under fire conditions, Journal of Structural Engineering 127 (10) (2001) 1214-1220.

[5] W. S. Toh, K. H Tan and T. C. Fung, Strength and stability of steel frames in fire: Rankine approach, Journal of Structural Engineering 127 (4) (2001) 461-469.

[6] W. S. Toh, K. H. Tan and T. C. Fung, Rankine approach for steel columns in fire: Numerical studies, Journal of
Constructional Steel Research 59 (2003) 315-334.

[7] W. Skowroński, Plastic load capacity and stability of frames in fire, Engineering Structures 19 (9) (1997) 764-771.

[8] W. Skowroński, Fire Safety of Metal Structures, Theory and Design Criteria, PWN, Warsaw, Poland 2004.

[9] EN 1993-1-2, Design of Steel Structures-Part 1-2: General Rules, Structural Fire Design, 2005. 